\title{
Effect of Operating Light Exposure on Enamel Bonding Performances of Universal Adhesives
}

\author{
Muhammet Kerim AYAR $^{1 *}$, Fatih ERDEMIR ${ }^{2}$ \\ ${ }^{1}$ Department of Restorative Dentistry, Faculty of Dentistry, Usak University, Usak, 64200, Turkey \\ ${ }^{2}$ Department of Metallurgical and Materials Engineering, Faculty of Engineering, Karadeniz Technical University, 61000, \\ Turkey \\ crossref http://dx.doi.org/10.5755/j01.ms.26.3.22863
}

Received 02 March 2019; accepted 10 July 2019

\begin{abstract}
The present study evaluated the effect of operating light exposure conditions on the shear bond strength (SBS) of composite resin bonded to enamel with three different universal adhesives used in self-etching mode. Tested adhesives were Single Bond Universal (SBU), G-Premio Bond (GB) and Nova Compo-B Plus (NB) and tested illumination conditions were ambient light, 22.000 lux, and 8.000 lux. Ninety bovine incisors were used for SBS test $(n=10)$. Effect of operating light exposure on the fluidity of adhesives was assessed. Resin-enamel interfaces were also evaluated under a scanning electron microscope. Two-way analysis of variance showed that material had a significant effect on SBS $(p<0.000)$ unlike the illumination condition $(p=0.459)$. GB provided significantly lower bond strength than those of SBU and NB $(p<0.05)$. High power operating light likely reduces the fluidity of all adhesives. Thus, it could be recommended that clinicians would prefer to use the tested resin adhesive systems at an illuminance of 8.000 lux.

Keywords: adhesive, dental materials, enamel, illumination, shear bond strength.
\end{abstract}

\section{INTRODUCTION}

Visible light-cured resin-based restorative materials currently have become almost a standard of care for the restoration of decayed teeth due to their enhanced aesthetics, superior handling characteristics and being mercury-free properties. Although these materials are generally accepted as being cured only by the use of light curing devices, they have a limited working time when exposed to ambient light and specifically operating light [1-4].

Resin restorative materials should be used necessarily with resin adhesive systems to achieve long-lasting bonding with tooth structures and sealing $[5,6]$. Resin adhesive systems are basically visible light-cured solvated resin monomer blends which have similar monomer chemistry with those of visible light-cured resin composites [7, 8]. They deploy photoinitiator systems within only bonding agents or within both of primers and bonding agents, making them sensitive to the ambient light and operating lights potentially, similarly with resin composite restoratives [8].

The essential function of resin-based adhesives is the bonding of the resin composite restorative materials to enamel and dentin long lastingly [5, 6, 9]. To achieve this properly, adhesive systems should perform some fundamental process such as etching, priming, and bonding. Some of the adhesive systems on the market establish these functions by only one complicated resin monomer-solventphotoinitiator blend in one bottle [5], while some adhesive systems contain multiple solutions in separated bottles which each one is decided to perform one or two fundamental functions under clinical conditions [7-9]. First mentioned adhesives are called as one bottle one-step so- called simplified resin adhesives, latter adhesives are called was three-step or two-step resin adhesive systems.

It has been widely accepted that simplification of application of resin adhesives resulted in very complicated resin blends in nature [10]. With the simplified resin adhesive systems, one drop adhesive solution is placed onto surfaces for a certain time i.e. 10-20 seconds to etch and hybridize enamel and dentin surfaces simultaneously, then solvent contents are evaporated by strong air pressure before their photo-polymerization by light curing device. Before photo-polymerization, the adhesive solution should etch and hybridize enough to achieve long-lasting bonding between resin composite and tooth structure [10]. If ambient light or operating lights would result in premature curing for adhesive resin before a required time to perform enough etching and priming, their immediate bonding performances would be impaired potentially. Previous studies reported that shortened application time of self-etch adhesives reduces enamel bond strength significantly $[11,12]$. This would also be critical specifically for bonding to enamel using self-etch adhesives. Because greater mineral content of enamel makes this substrate challenging for bonding of self-etch adhesives to enamel [13, 14].

However, there is limited knowledge about this issue in the literature. Some of the resin adhesive system manufacturers gave information about keeping away operating light from resin adhesive during the application, while others did not mention this issue in their instructions for use of their products. Nevertheless, as resin adhesives are potentially sensitive to ambient and operating lights because of their similar chemistry with resin composites, the effect of ambient and operating lights on bond strength of

*Corresponding author. Tel.: +276-22-12231; fax: +276-22-12232.

E-mail address: muhammet.ayar@usak.edu.tr (M.K. Ayar) 
universal adhesives to enamel should be investigated. Therefore, the null hypothesis that universal adhesives are able to bond to enamel regardless of different light conditions was tested in the present study.

\section{EXPERIMENTAL DETAILS}

Effects of operating light conditions on enamel shear bond strengths (SBS) of three different universal adhesives used in the self-etch mode were assessed. Tested parameters were (1) operating light conditions (ambient light -no operating light-, 22.000 lux operating light, 8.000 lux operating light), and (2) adhesives (Single Bond Universal, 3M Deutschland GmbH, Neuss, Germany; G-Premio Bond, GC Corporation, Tokyo; Nova Compo-B Plus, Imicryl, Konya, Turkey) (Table 1). Enamel shear bond strength was a dependent variable. Combinations of these parameters resulted in a total of 9 groups for the dependent variable $(n=10)$. Also, the effects of light conditions on adhesive fluidity and micromorphology of resin-enamel interfaces were evaluated under scanning electron microscopy.

Ninety bovine incisors extracted from 2-3 yearsold cattle were used as an enamel substitute for human teeth $[15,16]$. Teeth were stored in $1 \%$ Chloramine-T solution $4{ }^{\circ} \mathrm{C}$ prior to using. Crowns of teeth were separated from the roots by means of a diamond saw under water-cooling. Labial surfaces of crowns were flattened by 320-grit silicon carbide (SiC) abrasive papers underwater by hand. Then, crowns were embedded in self-curing acrylic resin, individually. Enamel surfaces were further polished with 600-grit SiC papers for 60 seconds to create standardized smear layer just prior to bonding procedures. Samples were randomly divided into according to adhesive systems and light conditions as following:

Ambient light: under this light condition, whole adhesive application procedures were performed under ambient light condition, no operating light was used. Each adhesive was applied to the enamel surfaces according to the manufacturer's instructions for use, respectively (Table 1). Light emitting diode (LED) curing device was used to polymerize adhesive (Mini Led Satelec, Acteon Group, Merignac, France; light intensity > $1200 \mathrm{~mW} / \mathrm{cm}^{2}$; wavelength, $420 \mathrm{~nm}-480 \mathrm{~nm})$.

Operating light exposure (22.000 lux): whole adhesive application procedures were performed under high power operating light exposure. Power of the operating light was 22.000 lux according to manufacturer's information (Sirona Intego dental unit, Sirona Dental Systems GmbH, Bensheim, Germany). The range of color temperature of the visible light emitted from operating light unit was $5700 \mathrm{~K}-6900 \mathrm{~K}$ according to the manufacturer. All bonding procedures in all teeth of this condition were performed at the fixed distance $(30 \mathrm{~cm})$ and perpendicular to the operating light. The same LED curing unit was used to polymerize adhesive.

Operating light exposure (8.000 lux): whole adhesive application procedures were performed under low power operating light exposure. Power of the operating light was 8.000 lux according to manufacturer's information (Sirona Intego dental unit, Sirona Dental Systems GmbH, Bensheim, Germany). All bonding procedures in all teeth of this condition were performed at the fixed distance $(30 \mathrm{~cm})$ and perpendicular to the operating light. LED curing unit was used to polymerize adhesive.

Following adhesive applications, a two-piece removable plexiglass mold was fixed on the surface, giving a cylindrical cavity $4 \mathrm{~mm}$ in height and $3 \mathrm{~mm}$ in diameter. Each adhesive system was used with the respective composite from the same manufacturer (Table 1). Resin composites were placed into the cavities incrementally. Each increment was polymerized for $20 \mathrm{~s}$ by using LED curing device (Mini Led Satelec, Acteon Group, Merignac, France; light intensity $>1200 \mathrm{~mW} / \mathrm{cm}^{2}$ ). Bonded teeth were immersed in water for $24-\mathrm{h}$ prior to shear bond strength tests. Specimens were loaded in shear mode until fracture happened with the use of universal testing machine (Mod Dental Mic-101, Esetron Mechatronic, Ankara, Turkey) at crosshead speed of $0.5 \mathrm{~mm} / \mathrm{min}$ (Fig. 1). The SBS (in megapascal, $\mathrm{MPa}$ ) was calculated by dividing the maximum load by the cross-sectional area of the bonded surface. Fracture surfaces of the specimens were examined visually with light microscope at a standard $\times 40$ magnification to determine the failure region. Failure modes were divided into adhesive, cohesive and mixed failure.

The statistical evaluation of the failure mode distribution was performed with the use of the chi-squared test with contingency tables, at a significance level of $\%$. Nine bovine incisors were bonded in the same way as they were for the shear bond strength test.

Table 1. Materials used in the present study

\begin{tabular}{|c|c|c|}
\hline Adhesives & Chemical composition & Instructions for use \\
\hline $\begin{array}{l}\text { G-Premio Bond, GC Corporation, Tokyo, } \\
\text { Japan LOT: } 1603021\end{array}$ & $\begin{array}{l}\text { 10-MDP, 4-META, } \\
\text { 10-methacryloyloxydecyl } \\
\text { dihydrogen thiophosphate, } \\
\text { methacrylic acid ester, acetone, water, } \\
\text { initiators }\end{array}$ & $\begin{array}{l}\text { 1. Apply the adhesive to the prepared tooth } \\
\text { and leave it in for } 10 \mathrm{~s} \text {. } \\
\text { 2. Dry thoroughly for } 5 \mathrm{~s} \text { with maximum air } \\
\text { pressure. } \\
\text { 3. Light cure for } 10 \mathrm{~s} \text {. }\end{array}$ \\
\hline $\begin{array}{l}\text { 3M ESPE Single Bond Universal, 3M } \\
\text { Deutschland GmbH, Neuss, Germany } \\
\text { LOT: } 620316\end{array}$ & $\begin{array}{l}\text { 10-MDP, dimethacrylate resins, } \\
\text { HEMA, Vitrebond copolymer, filler, } \\
\text { ethanol, water, initiators, silane }\end{array}$ & $\begin{array}{l}\text { 1. Apply the adhesive to the prepared tooth } \\
\text { and rub it in for } 20 \mathrm{~s} \text {. } \\
\text { 2. Gently air dry the adhesive for } \\
\text { approximately } 5 \mathrm{~s} \text {. } \\
\text { 3. Light cure for } 10 \mathrm{~s} \text {. }\end{array}$ \\
\hline $\begin{array}{l}\text { Nova Compo-B Plus, Imicryl, Konya, } \\
\text { Turkey } \\
\text { LOT: } 17024\end{array}$ & $\begin{array}{l}\text { Bis-GMA, HEMA, ethanol, 10-MDP, } \\
\text { 4-META, silanated nano silica, } \\
\text { initiators, water }\end{array}$ & $\begin{array}{l}\text { 1. Apply the adhesive to the prepared tooth } \\
\text { and agitate it in for } 20 \mathrm{~s} \text {. } \\
\text { 2. Gently air dry the adhesive for } \\
\text { approximately } 5 \mathrm{~s} \text {. } \\
\text { 3. Light cure for } 10 \mathrm{~s} \text {. }\end{array}$ \\
\hline
\end{tabular}




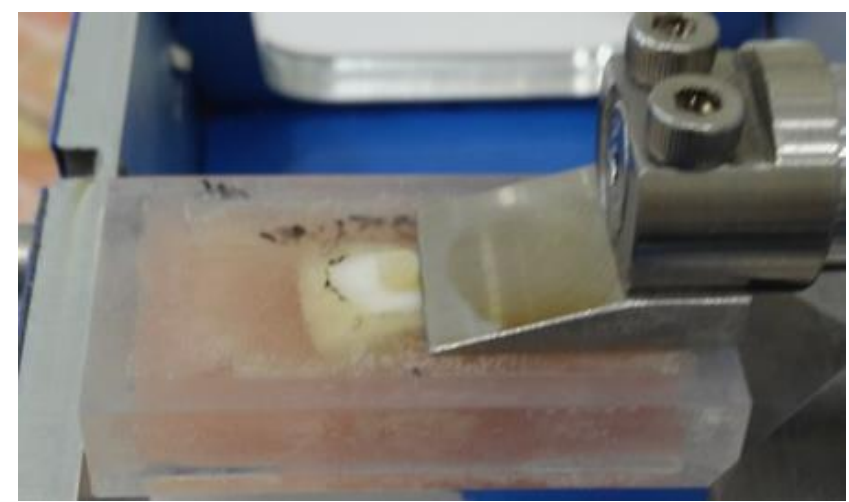

Fig. 1. Presentive picture shows the operation of shear bond strength test

Then, the teeth were sectioned perpendicular to the resin-enamel interface in order to obtain two parallel $2 \mathrm{~mm}$ thick resin-dentin slabs. The exposed interfaces were subsequently polished with \#800, 1000 and $1500 \mathrm{SiC}$ papers under running water. Specimens were dried at room temperature for 24-hours. All specimens were then gold sputter coated and the surfaces were examined under a SEM with an operating voltage of $15 \mathrm{kV}$ (SEM, Zeiss EVO LS10, Bruker, Bremen, Germany). The SEM images were captured at the magnification of $5000 \times$.

The fluidity of each adhesive under different light conditions were also visualized by placing aliquot amounts of each adhesive samples on glass slides. The slides were arranged in an upright position. Before and after exposing slides to each light condition for 20 seconds, photographs were taken.

Means and standard deviations of the shear bond strength values of adhesive resins were calculated, respectively. For checking normal distribution and homogeneity for variances among the groups, a Kolmogorov-Smirnov test, and a Levene Test were applied to data, respectively. This primary analysis showed that normal distributions and homogeneity of variances existed for all groups. Thus, parametric tests were applied to data. A two-way analysis of variation (ANOVA) was conducted to determine the effects of the light condition, material and the interaction of these two factors on the dependent variable (Shear bond strength) [12]. Then, one-way ANOVA and the Least Significant Difference (LSD) tests were used for post-hoc multiple comparisons. All statistical analyses were performed using SPSS 13 for Windows (SPSS Inc., Chicago, IL, USA) at a $5 \%$ level of significance.

\section{RESULTS AND DISCUSSION}

Enamel shear bond strengths of all groups are presented in Table 2. Two-way ANOVA showed that enamel shear bond strength means were significantly affected by the material $(p<0.000)$ but not by the light condition $(p=0.459)$, and there was no significant interaction between the material and light condition $(p=0.775)$. Single Bond Universal provided significantly higher enamel bond strengths than those of G-Premio Bond under all light conditions, however, Nova Compo-B Plus showed similar bond strengths with those of Single Bond Universal (Table 2). Failure mode analysis revealed a higher incidence of adhesive failure for G-Premio Bond groups and Nova Compo-B Plus groups, while incidences of cohesive failures were higher for Single Bond Universal groups with no significance according to the chi-squared test (Table 2).

The different fluidity characteristics of each adhesive under different light conditions are shown in Fig. 2. Without operating light exposure and exposing light exposure of 8.000 lux showed no alteration in adhesive fluidity for all adhesives. Noticeable movements of the resin drop downward were seen (Fig. $2 \mathrm{a}$ and b). However, the situation changed after exposing adhesives to 22.000 lux operating light, small dents were detected only superficially, for Single Bond Universal and G-Premio bond adhesives demonstrating increased viscosity. However, noticeable lowering in the movement of resin drop of Nova Compo-B Plus downwards was seen under this light condition (Fig. 2 c) SEM observations of resin-enamel interfaces showed that all self-etch adhesives produced adhesive layers with similar thickness, regardless of operating light conditions at the resin-enamel interfaces (Fig. 3).

To analyze if operating light exposures with different illumination powers have an effect on enamel bonding, three commercially available universal adhesives were studied in the present study. These resin adhesive systems are called as universal adhesives, as manufacturers claim that these adhesives could be used in total-etching, self-etching and selective etching modes for enamel and dentin bonding [17].

The quality of the treatment depends on the visual acuity of the dentist. For this, the operation area must be adequately illuminated by the operating light. However, the intensity and duration of the operational light may cause premature polymerization of the resin-based materials. For this reason, some dentists prefer to turn off operating light and only use only ambient light conditions during manipulation of resin-based materials.

Table 2. Enamel shear bond strength means (MPa), and failure modes of each adhesives under different light conditions

\begin{tabular}{|c|c|c|c|c|c|c|c|c|c|c|c|c|}
\hline \multirow{3}{*}{$\begin{array}{l}\begin{array}{l}\text { Operating light } \\
\text { conditions }\end{array} \\
\text { Adhesives }\end{array}$} & \multicolumn{4}{|c|}{ Ambient light (operating light is off) } & \multicolumn{4}{|c|}{$22.000 \operatorname{lux}$} & \multicolumn{4}{|c|}{$8.000 \operatorname{lux}$} \\
\hline & \multirow{2}{*}{ Bond strength, $\mathrm{MPa}$} & \multicolumn{3}{|c|}{$\begin{array}{l}\text { Failure modes, } \\
\%\end{array}$} & \multirow{2}{*}{$\begin{array}{l}\text { Bond strength, } \\
\mathrm{MPa}\end{array}$} & \multicolumn{3}{|c|}{$\begin{array}{l}\text { Failure modes, } \\
\%\end{array}$} & \multirow{2}{*}{$\begin{array}{l}\text { Bond strength, } \\
\mathrm{MPa}\end{array}$} & \multicolumn{3}{|c|}{ Failure modes, $\%$} \\
\hline & & $\mathrm{A}$ & $\mathrm{M}$ & $\mathrm{C}$ & & $\mathrm{A}$ & $\mathrm{M}$ & $\mathrm{C}$ & & $\mathrm{A}$ & $\mathrm{M}$ & $\mathrm{C}$ \\
\hline Single bond universal & $14.44 \pm 4.3^{\mathrm{a}}$ & 40 & 20 & 40 & $13.22 \pm 3.5^{\mathrm{a}}$ & 40 & 30 & 30 & $13.97 \pm 4.0^{\mathrm{a}}$ & 50 & 30 & 20 \\
\hline G-Premio bond & $9.78 \pm 1.9^{b}$ & 80 & 20 & - & $7.88 \pm 1.9^{b}$ & 70 & 30 & 10 & $8.91 \pm 2.1^{\mathrm{b}}$ & 70 & 30 & - \\
\hline Nova compo-B plus & $12.58 \pm 2.4^{\mathrm{a}}$ & 70 & 20 & 10 & $12.9 \pm 0.8^{\mathrm{a}}$ & 50 & 40 & 10 & $12.15 \pm 1.9^{\mathrm{a}}$ & 60 & 30 & 10 \\
\hline
\end{tabular}




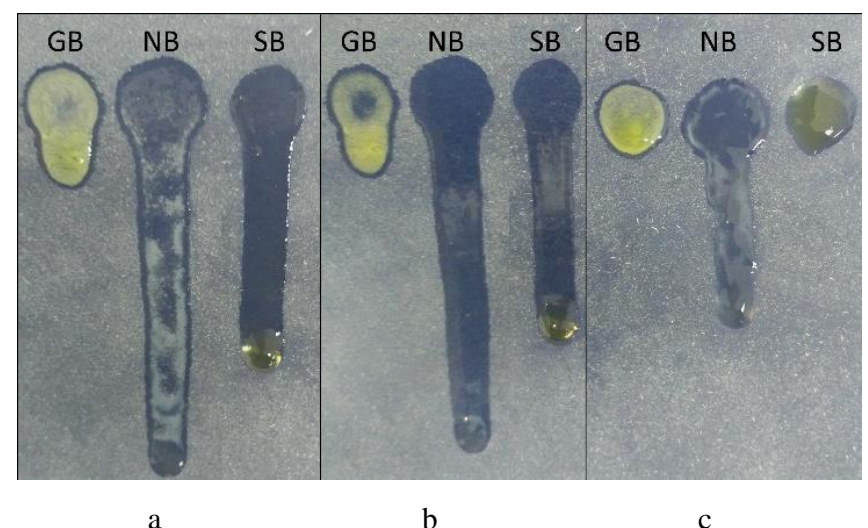

Fig. 2. Fluidity characteristics of each adhesive under different light conditions shown on upright-positioned glass slides: $\mathrm{a}$-ambient light condition; $\mathrm{b}$-operating light (8.000 lux); $\mathrm{c}$-operating light (22.000 lux). (GB: G-Premio Bond, NB: Nova Compo-B Plus, SB: Single Bond Universal)

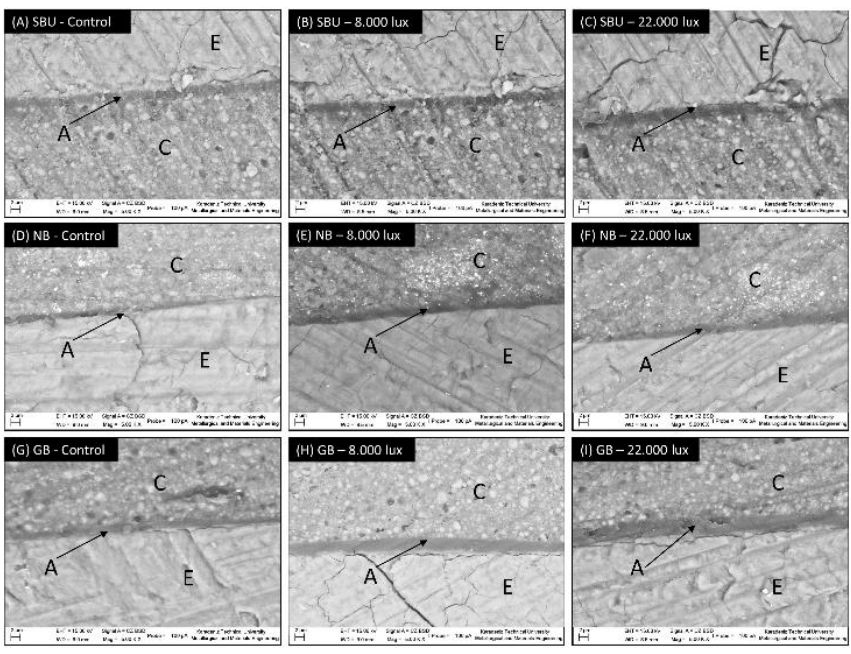

Fig. 3. SEM micrographs show the interfacial morphology of enamel bonded with Single Bond Universal, Nova CompoB Plus and G-Premio Bond at different illumination conditions, ambient light (Control), illumination of 8.000 lux and illumination of 22.000 lux, respectively (A-I). (C-composite, $\mathrm{E}$-enamel, $\mathrm{A}$-adhesive layer, $\mathrm{GB}-\mathrm{G}$ Premio Bond, NB-Nova Compo-B Plus, SB-Single Bond Universal)

International standards organization (ISO) has also set a standard. Current ISO standard for restorative materials and luting cements claims that working time of any restorative material and luting cements should be at least 90 seconds when exposed to operating light of 8.000 lux [18]. However, current advanced dental units deploy operating lights with 22.000 , even 25.000 lux powers for enhanced illumination of the operating area within the oral cavity. Therefore, uncured resin adhesive systems were exposed to operating light of 22.000 lux and 8.000 lux, respectively in the present study.

Resin bonding to enamel and dentin with simplified adhesive systems should be considered as highly techniquesensitive clinical procedures since a number of factors including material $[17,19]$, evaporation of solvents and water [17], type of adhesive application [6, 17, 20], application time [12] affect their bonding performances. It seems meaningful that sensitivity of light-cured resin adhesive to operating light exposure would be another potential factor would affect bonding performances of selfetch adhesive, since effects of potentially premature polymerization of adhesive resin due to exposing operating light have not well-studied in the literature. In the present study, it was found that enamel bond strength was not dependent on operating light conditions, rather it was dependent on adhesive brand. Therefore, the null hypothesis that universal adhesives are able to bond to enamel regardless of different light conditions was failed to be rejected. However, it was found that adhesive fluidity was significantly affected by both adhesive brand and illumination conditions.

Several explanations could account why bonding abilities of universal adhesives to enamel did not exhibit sensitivity to both operating light conditions. One possible explanation would be that, exposing to operating light would pre-polymerize only the outer surface of the uncured adhesive after completely spreading through enamel surface, thus inner surface (enamel front) of uncured adhesive layer would able to continue to etch enamel for sufficient bond strength during application of resin adhesive. This also might explain why the illumination of 22.000 lux reduced the fluidity of all adhesive systems tested in this study (Fig. 2). The pre-polymerized outer surface of adhesive would prevent flowing of inner uncured resin adhesive.

One consideration could be bearing in mind about the effect of illumination during application of resin adhesive would be that if pre-polymerization of adhesive due illumination would affect the evaporation of solvent within adhesive if it reduces the fluidity of adhesive [21]. To address this, interfacial morphology of resin-enamel interfaces and the thickness of adhesive layers were examined under scanning electron microscopy. Findings of SEM evaluation suggested that uniformness and thickness of adhesive layer for each adhesive did not depend on illumination conditions. Single Bond Universal and Nova Compo-B Plus adhesives were applied to enamel surface with agitation according to manufacturers' instructions. For G-Premio Bond, the adhesive was not applied with agitation, but adhesive dried with maximum air pressure. Possibly, active application of Single Bond Universal and Nova Compo-B Plus and strong air blowing of G-Premio Bond would prevent or remove of pre-polymerized upper adhesive layer prior to being cured by light curing device. However, it should be addressed that flat enamel surfaces were used in this in-vitro study, unlike clinical conditions including irregular cavity surfaces and configurations.

In the present investigation, bovine teeth were used as a substitute of human enamel. Bovine incisors are used for many reasons. In fact, it is easier to obtain a sufficient number of bovine teeth than human teeth. Moreover, the bigger surface area of bovine lower incisors allows correct preparation of a standardized bonding area. Finally, bovine teeth, derived from animals of similar genetic lineage and dietary environment, might show higher homogeneity of mineral composition than different human teeth, which are collected from various donators with diverse dietary or fluoride supplementation $[22,23]$. 


\section{CONCLUSIONS}

Within the limits of the present in-vitro study, considering operation light conditions and the related variables including, enamel bond strength, fluidity, and interfacial morphology of the tested universal adhesives, it can be concluded that

1. Enamel bond strength and interfacial morphology of the tested universal adhesive could not be significantly influenced by operating light conditions.

2. Illumination of operation area with a light power of 8.000 lux during adhesive application procedures resulted in similar fluidity with ambition light condition, while illumination of 22.000 lux reduced the fluidity of all tested universal adhesives. It seems that fluidity of the tested adhesives could be influenced by operating light conditions.

3. The illumination of 8.000 lux (low power operating light) could be preferred during resin adhesive system application because it does not reduce the fluidity of the tested universal adhesives while maintaining the dentist's visual acuity.

\section{REFERENCES}

1. Dionysopoulos, P., Watts, D. Sensitivity to Ambient Light of Visible Light-Cured Composites Journal of Oral Rehabilitation 17 1990: pp. 9-13. https://doi.org/10.1111/j.1365-2842.1990.tb01388.x

2. Lane, D.A., Watts, D.C., Wilson, N.H. Ambient Light Working Times of Visible Light-Cured Restorative Materials. Does the ISO Standard Reflect Clinical Reality? Dental Materials 14 1998: pp. 353-357. https://doi.org/10.1016/S0109-5641(99)00005-6

3. Malhotra, N., Kundabala, M. Light-Curing Considerations for Resin-Based Composite Materials: A Review. Part II Compendium of Continuing Education in Dentistry 31 2010: pp. $584-588$.

4. Merdji, A., Della, N., Benaissa, A., Bouiadjra, B.A.B., Serier, B., Mootanah, R., Muslih, I., Mukdadi, O.M. Numerical Analysis of Dental Caries Effect on The Biomechanical Behavior of The Periodontal System Journal of Nanotechnology in Engineering and Medicine 6 2015: pp. 031004-031011.

https://doi.org/10.1115/1.4032689

5. Araújo, C.T.P., Prieto, L.T., Costa, D.C., Bosso, M.A., Coppini, E.K., Dias, C.T.S., Paulillo, L.A.M.S. Active Application of Primer Acid on Acid-Treated Enamel: Influence on The Bond Effectiveness of Self-Etch Adhesives Systems Microscopy Research and Technique 80 2017: pp. $943-949$.

https://doi.org/10.1002/jemt.22887

6. Nagpal, R., Sharma, P., Manuja, N., $\quad$ Tyagi, S.P., Singh, U.P., Singh, S., Singh, P. Influence of Double Application Technique on The Bonding Effectiveness of SelfEtch Adhesive Systems Microscopy Research and Technique 78 2015: pp. 489-494. https://doi.org/10.1002/jemt.22499

7. Moszner, N., Salz, U., Zimmermann, J. Chemical Aspects of Self-Etching Enamel-Dentin Adhesives: A Systematic Review Dental Materials 21 2005: pp. 895-910. https://doi.org/10.1016/j.dental.2005.05.001

8. Van Landuyt, K.L., Snauwaert, J., De Munck, J., Peumans, M., $\quad$ Yoshida, Y., $\quad$ Poitevin, A., Van Meerbeek, B. Systematic Review of the Chemical
Composition of Contemporary Dental Adhesives Biomaterials 28 2007: pp. 3757-3785.

https://doi.org/10.1016/j.biomaterials.2007.04.044

9. Freitas, S.A.A.D., Lanza, M.D.S., Carneiro, K.K., Loguercio, A.D., Bauer, J. Evaluation of Degree of Conversion, Microtensile Bond Strength and Mechanical Properties of Three Etch-And-Rinse Dental Adhesives Polímeros 27 2017: pp. 230-236. https://doi.org/10.1590/0104-1428.02316

10. Van Landuyt, K., De Munck, J., Snauwaert, J., Coutinho, E., Poitevin, A., Yoshida, Y., Lambrechts, P. Monomer-Solvent Phase Separation in One-Step Self-Etch Adhesives Journal of Dental Research 84 2005: pp. $183-188$. https://doi.org/10.1177/154405910508400214

11. Hayakawa, T., Kikutake, K., Nemoto, K. Influence of SelfEtching Primer Treatment on The Adhesion of Resin Composite to Polished Dentin and Enamel Dental Materials 14 1998: pp. 99-105. https://doi.org/10.1016/S0109-5641(98)00015-3

12. Perdigao, J., Gomes, G., Lopes, M.M. Influence of Conditioning Time on Enamel Adhesion Quintessence International 37 2006: pp. 35-41.

13. De Munck, J., Van Meerbeek, B., Satoshi, I., Vargas, M., Yoshida, Y., Armstrong, S., Vanherle, G. Microtensile Bond Strengths of One-and Two-Step Self-Etch Adhesives to Bur-Cut Enamel and Dentin American Journal of Dentistry 16 2003: pp. 414-420.

14. Mine, A., De Munck, J., Cardoso, M.V., Van Landuyt, K.L., $\quad$ Poitevin, A., $\quad$ Kuboki, T., Van Meerbeek, B. Bonding Effectiveness of Two Contemporary Self-Etch Adhesives to Enamel and Dentin Journal of Dentistry 37 2009: pp. 872-883. https://doi.org/10.1016/j.jdent.2009.06.020

15. Nakamichi, I., Iwaku, M., Fusayama, T. Bovine Teeth as Possible Substitutes in the Adhesion Test Journal of Dental Research 62 1983: pp. 1076-1081. https://doi.org/10.1177/00220345830620101501

16. Schilke, R., Bauss, O., $\quad$ Lisson, J., $\quad$ Schuckar, M., Geurtsen, W. Bovine Dentin as A Substitute for Human Dentin in Shear Bond Strength Measurements American Journal of Dentistry 12 1999: pp. $92-96$.

17. Fu, J., Saikaew, P., Kawano, S., Carvalho, R.M., Hannig, M., Sano, H., Selimovic, D. Effect of Air-Blowing Duration on the Bond Strength of Current One-Step Adhesives to Dentin Dental Materials 33 2017: pp. $895-903$. https://doi.org/10.1016/j.dental.2017.03.015

18. International Organization for Standardization. ISO 4049 Dentistry--Polymer-based Restorative Materials ISO 2009.

19. Manuja, N., Nagpal, R. Resin-Tooth Interfacial Morphology and Sealing Ability of One-Step Self-Etch Adhesives: Microleakage and SEM Study Microscopy Research and Technique 75 2012: pp. 903-909. https://doi.org/10.1002/jemt.22011

20. Miyazaki, M., Hinoura, K., Honjo, G., Onose, H. Effect of Self-Etching Primer Application Method on Enamel Bond Strength American Journal of Dentistry 15 2002: pp. $412-416$

21. Yiu, C.K., Pashley, E.L., Hiraishi, N., King, N.M., Goracci, C., Ferrari, M., Tay, F.R. Solvent and Water Retention in Dental Adhesive Blends after Evaporation Biomaterials 26 2005: pp. 6863-6872. https://doi.org/10.1016/j.biomaterials.2005.05.011 
22. Hanabusa, M., Mine, A., Kuboki, T., Momoi, Y., Van Ende, A., Van Meerbeek, B., De Munck, J. Bonding Effectiveness of a New 'Multi-Mode' Adhesive to Enamel and Dentine Journal of Dentistry 40 2012: pp. 475-484. https://doi.org/10.1016/j.jdent.2012.02.012
23. Poggio, C., Beltrami, R., Colombo, M., Chiesa, M., Scribante, A. Influence of Dentin Pretreatment on Bond Strength of Universal Adhesives Acta Odontologica Scandinavica 3 2017: pp. 30-35.

https://doi.org/10.1080/23337931.2017.1305273 